\title{
(2) OPEN ACCESS \\ Household and area-level social determinants of multimorbidity: a systematic review
}

\author{
Elizabeth Ingram (1), ${ }^{1}$ Sarah Ledden, ${ }^{2}$ Sarah Beardon, ${ }^{1}$ Manuel Gomes, ${ }^{1}$ Sue Hogarth, ${ }^{3}$ \\ Helen McDonald, ${ }^{4}$ David P Osborn, ${ }^{2,5}$ Jessica Sheringham ${ }^{1}$
}

\begin{abstract}
- Supplemental material is published online only. To view please visit the journal online (http://dx.doi.org/10.1136/ jech-2020-214691).
\end{abstract}

For numbered affiliations see end of article.

\section{Correspondence to}

Elizabeth Ingram, Department of Applied Health Research, Institute of Epidemiology and Health

Care, University College London, London, UK;

e.ingram.17@ucl.ac.uk

Received 5 June 2020 Revised 16 September 2020

Accepted 14 October 2020

Published Online First

6 November 2020
ABSTRACT

Background No clear synthesis of evidence examining household and area-level social determinants of multimorbidity exists. This study aimed to systematically review the existing literature on associations between household and area-level social determinants of health (SDOH) and multimorbidity prevalence or incidence in the general population.

Methods Six databases (MedLine, EMBASE, PsychINFO, Web of Science, CINAHL Plus and Scopus) were searched. The search was limited to peer-reviewed studies conducted in high-income countries and published in English between 2010 and 2019. A second reviewer screened all titles with abstracts and a subset of full texts. Study quality was assessed and protocol pre-registered (CRD42019135281)

Results 41 studies spanning North America, Europe and Australasia were included. Household income and arealevel deprivation were the most explored with fairly consistent findings. The odds of multimorbidity were up to 4.4 times higher for participants with the lowest level of income compared with the highest level. Those living in the most deprived areas had the highest prevalence or incidence of multimorbidity (pooled OR 1.42, 95\% Cl 1.41 to 1.42). Associations between deprivation and multimorbidity differed by age and multimorbidity type. Findings from the few studies investigating household tenure, household composition and area-level rurality were mixed and contradictory; homeownership and rurality were associated with increased and decreased multimorbidity, while living alone was found to be associated with a higher risk of multimorbidity and not associated.

Conclusion Improving our understanding of broader social determinants of multimorbidity — particularly at the household level_could help inform strategies to tackle multimorbidity.

\section{INTRODUCTION}

Multimorbidity is one of the greatest challenges for health and care systems worldwide. ${ }^{1}$ Broadly defined as the co-occurrence of multiple chronic conditions within the same individual, ${ }^{2}$ multimorbidity is now the norm internationally, not the exception. ${ }^{3}$ Indeed, approximately one-third of UK primary care patients has two or more long-term conditions. ${ }^{4}$ This is projected to increase dramatically over the coming decade. ${ }^{5}$

Multimorbidity challenges health and care systems because care must, by definition, cross organisational and sectoral boundaries. ${ }^{6} 7$ This requires a radical shift in approach from a fragmented medical model of illness, centred on specific disorders, to a more holistic view of health. There are also calls for approaches focused on preventing multimorbidity or curtailing multimorbidity progression that could minimise the future burden on the system. ${ }^{1}$ Such shifts in approach require an understanding of the broader factors associated with multimorbidity.

While multimorbidity is often framed as a health issue, it is greatly influenced in extent and nature by social determinants of health $(\mathrm{SDoH})$. There are multiple conceptualisations of $\mathrm{SDoH}$; nevertheless, a hierarchical division of individual, household and area-level social factors is common to many. ${ }^{8-10}$ Individual social determinants (SD) of multimorbidity are well established-prevalence is greater among ethnic minorities and individuals with fewer educational qualifications. ${ }^{11-14}$ However, understanding of household and area-level SD is limited with most primary research focusing on area-level deprivation indices. At present, no clear synthesis of evidence examining household and area-level SD of multimorbidity exists. ${ }^{11} 15-18$

This review aimed to systematically identify, critically appraise and synthesise the existing literature on associations between household and area-level $\mathrm{SDoH}$ and multimorbidity prevalence or incidence in general populations of high-income countries (HICs). We also aimed to investigate how associations differ with age, gender and ethnicity. Better understanding of SD of multimorbidity could inform equitable prevention and intervention strategies.

\section{METHODS}

This review was conducted following PRISMA guidelines $^{19}$ and the protocol prospectively registered with PROSPERO (CRD42019135281).

\section{Eligibility criteria for inclusion}

Studies were included if in English, conducted in HICs and published between 1 January 2010 and 10 January 2019. The former date restriction coincides with the publication of The Marmot Report, which raised the profile of SDoH in England. ${ }^{20}$ We excluded studies conducted solely with institutionalised individuals as SD of multimorbidity may differ between institutional and community settings. ${ }^{21}$ We excluded studies conducted with solely young people ( $<18$ years) as prevalence is low for this group ${ }^{22}$ (table 1).

\section{Search strategy}

The following six databases were searched: MedLine, EMBASE, PsychINFO, Web of Science, CINAHL Plus and Scopus. Terms relating to multimorbidity, specific SDoH and household or area 
Table 1 Inclusion and exclusion criteria

\begin{tabular}{|c|c|c|}
\hline & Inclusion & Exclusion \\
\hline Population & $\begin{array}{l}\text { Participants from the general } \\
\text { population and assessed for the } \\
\text { presence of multiple chronic } \\
\text { conditions (multimorbidity). }\end{array}$ & $\begin{array}{l}\text { Participants initially selected } \\
\text { based on the presence of index } \\
\text { diseases (ie, studies of } \\
\text { comorbidity). Participants from } \\
\text { solely institutionalised care } \\
\text { settings (eg, nursing homes). } \\
\text { Participants solely young people } \\
\text { (age }<18 \text { years). }\end{array}$ \\
\hline Exposure & $\begin{array}{l}\text { Study exposure(s) included at least } \\
\text { one household or area-level SDoH } \\
\text { that aligns with factors from the } \\
\text { World Health Organisation (WHO) } \\
\text { Commission on SDoH (CSDH) } \\
\text { Framework } \\
\text { SDoH are 'causes of the causes' of } \\
\text { ill-health }{ }^{20} \text { (eg, household income } \\
\text { or area-level deprivation). }\end{array}$ & $\begin{array}{l}\text { Study exposure(s) include } \\
\text { individual SDoH only (eg, } \\
\text { ethnicity). Study exposure(s) are } \\
\text { direct "causes" of ill-health, such } \\
\text { as health behaviours (eg, } \\
\text { smoking), or are factors } \\
\text { associated with the health system } \\
\text { itself (eg, access to services). }\end{array}$ \\
\hline Comparator & $\begin{array}{l}\text { Study reports comparator group(s) } \\
\text { for SDoH exposure(s) (eg, what is } \\
\text { the prevalence of multimorbidity } \\
\text { for those in the lowest vs the } \\
\text { highest household income } \\
\text { groups). }\end{array}$ & $\begin{array}{l}\text { Study does not report } \\
\text { a comparator group for SDoH } \\
\text { exposure(s). }\end{array}$ \\
\hline Outcome & $\begin{array}{l}\text { Assess multimorbidity burden } \\
\text { (prevalence or incidence studies). }\end{array}$ & $\begin{array}{l}\text { Assignment to multimorbidity } \\
\text { patterns or trajectories. Measures } \\
\text { of multimorbidity severity (eg, } \\
\text { indices used weighted by disease } \\
\text { severity). }\end{array}$ \\
\hline $\begin{array}{l}\text { Study } \\
\text { design }\end{array}$ & $\begin{array}{l}\text { Peer-reviewed studies of } \\
\text { quantitative research designs } \\
\text { (cross-sectional and longitudinal). }\end{array}$ & $\begin{array}{l}\text { Systematic reviews, meta- } \\
\text { analyses and qualitative research } \\
\text { (citations of relevant reviews } \\
\text { searched). }\end{array}$ \\
\hline
\end{tabular}

were combined using Boolean language. We drew on published frameworks and previous literature to develop SDoH search terms. ${ }^{8-10}$ 24-27 Terms were initially developed in MedLine (online appendix 1) and adapted for each database. After the initial search, we added the MeSH term 'comorbidity' into our MedLine search to examine if any studies had been missed through excluding the term 'comorbidity' and its linguistic variations. A combination of forward and backward citation searching, and searching citations of relevant reviews, was used to identify further studies.

EI and SL independently screened titles and abstracts of all records from database searches. A third reviewer independently screened 100 randomly selected records. EI screened all full texts and SL a subset (20\%). All relevant data were extracted by EI using a pre-piloted form including study characteristics, definitions of exposures and outcomes, and findings (online appendix 2). The authors were contacted if data extraction was incomplete. Inter-rater reliability was calculated using Kappa statistics and differences resolved by discussion.

\section{Quality assessment}

Study quality was assessed within four domains: selection bias, information bias for exposure and outcome, and confounding (online appendix 3). Non-interventional studies are rarely at low overall risk of bias and reporting by domain allows comparison of the main sources of bias across studies. ${ }^{28}$ Each study was assigned high, medium, low or unclear ratings for each domain, to separate study quality from reporting quality. ${ }^{29}{ }^{30}$ Criteria were specific to the study and informed by existing checklists. ${ }^{28}{ }^{31}$ Risk of selection bias was assessed by comparing sample demographics to census data when possible. Studies where risk of bias was high across two or more domains were deemed low quality. Studies where risk of bias was mixed or medium across all domains were deemed moderate. Studies with a low risk of bias across two or more categories, with no high risk of bias across any domains, were deemed high quality. EI completed all assessments and SL a random subset (20\%). Quality assessments were used to provide insight into the overall quality of evidence in this field, rather than to exclude or rank studies. Assessments were also used to explore any associations between study results and quality assessments.

\section{Data synthesis}

Findings were narratively synthesised given the diverse exposures, outcomes and study methodologies. Studies were too heterogeneous to allow a meta-analysis of findings. Available data were pooled for studies investigating area-level deprivation to calculate overall multimorbidity prevalence in deprivation quintiles.

\section{RESULTS}

\section{Study selection and characteristics}

Forty-one studies were included (figure 1). Inter-rater reliability was good for title and abstract screening $(\kappa=0.71)$, and full-text $(\kappa=0.77){ }^{32}$

Online appendix 4 details key study characteristics. Studies were conducted in a range of countries, the most common being Canada, England and Spain. Sample sizes ranged from 232 to 13581 191. Twenty-five studies included participants from across the life-course, while nine focused on adults aged 50 and over. Household SDoH included measures of household income, tenure and composition, self-reported by participants in all studies. Area-level SDoH included measures of socioeconomic deprivation and rurality, the former measured using validated indices (16/17 studies) and polling data (one study).

\section{Defining and measuring multimorbidity}

Most studies (34/41) defined multimorbidity as two or more conditions taken from a pre-specified list of 'long-term' or 'chronic' conditions. Eight also used three or more conditions as the cut-off and seven studies used a count of conditions as one outcome. Six studies examined if associations differed with multimorbidity type: multimorbidity specifically comprising physical and mental health conditions (physical-mental multimorbidity), physical conditions (physical-only multimorbidity) and mental health conditions (mental-only multimorbidity).

Across the 41 studies, the number of conditions included on the pre-specified list ranged from five conditions to 146 diagnostic clusters defined using O'Halloran's criteria for chronicity. ${ }^{33}$ Thirty-six of 41 studies included a mix of chronic physical and mental health conditions, ${ }^{422}$ 34-67 while four included physical conditions only ${ }^{68-71}$ One study was unclear about the conditions included. ${ }^{72}$ To determine the presence of conditions, 17 studies used self-reported participant data, 17 screened electronic health records (EHRs), and six used a combination of the two. One study did not detail how they identified multimorbidity presence $^{70}$ (online appendix 4).

\section{Study results}

Household income $(n=15)$ : Thirteen studies consistently found multimorbidity prevalence or incidence was markedly and negatively associated with household income and, of all SDoH investigated, associations were consistently strongest for household 


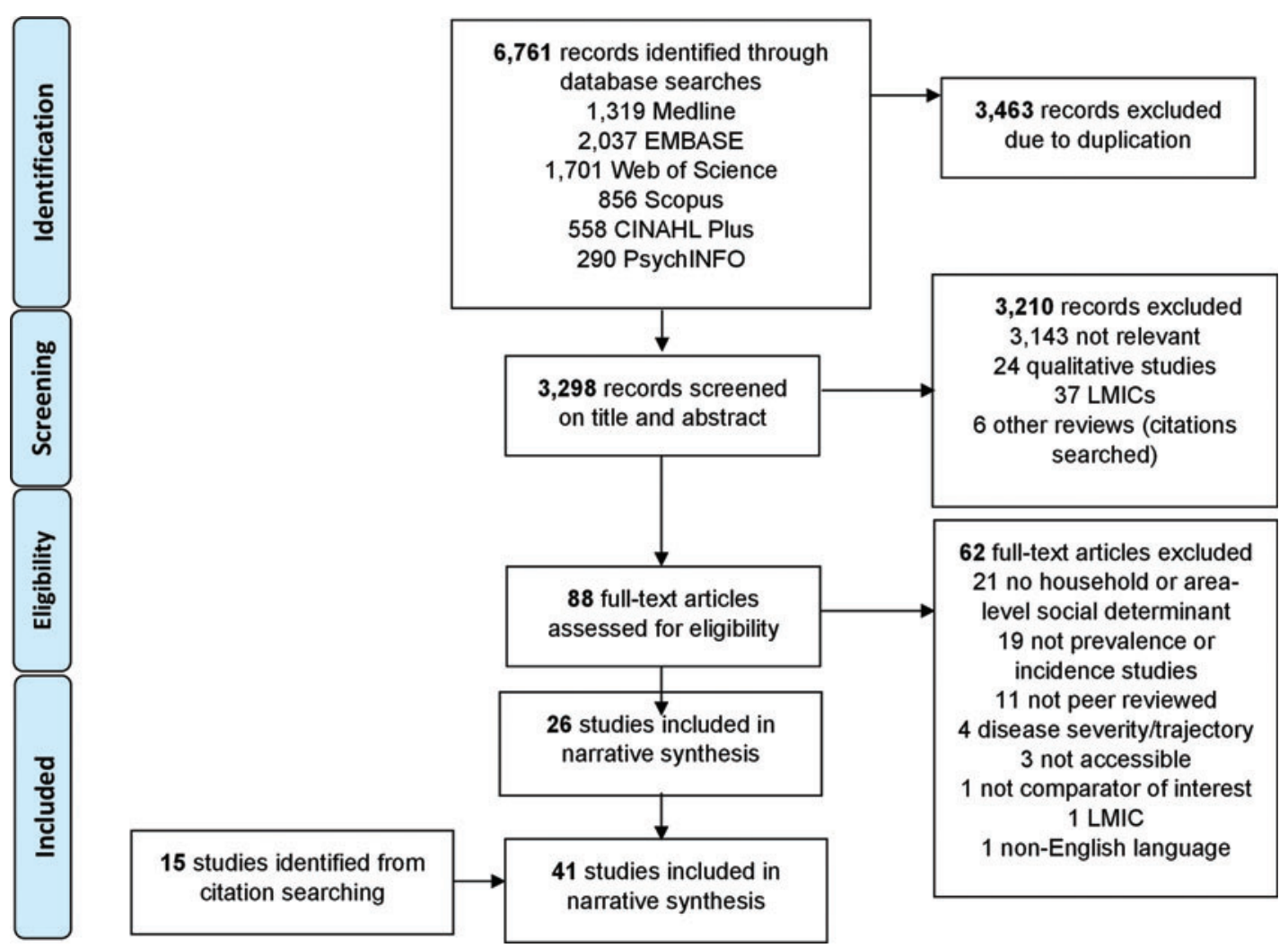

Figure 1 PRISMA flow diagram.

income. $^{39-41} 43 \quad 48 \quad 53 \quad 60-62 \quad 65 \quad 66 \quad 68 \quad 69$ Higher quality studies reported comparatively small estimated effect sizes, for example, Agborsangaya et al reported that an annual household income $<\$ 30000 \mathrm{CAD}$ was associated with a 2.39-fold increase in multimorbidity prevalence (95\% CI 1.72 to 3.33 ) compared with $\geq \$ 100000 \mathrm{CAD}$, after multiple adjustments. ${ }^{62}$ In contrast, Roberts et al-a lower quality study-reported chances of multimorbidity 4.4 times higher for participants with the lowest level of income compared with the highest in multivariate analyses (OR 4.4, 95\% CI 3.6 to 5.5). ${ }^{53}$

Two further studies - of low and moderate quality, respectivelyexamined problems managing household income and reported mixed results. ${ }^{5259}$ Verest et al reported those with 'lots of problems' were over 5 times likely to have multimorbidity compared with those with 'no problems' (OR 5.36, 95\% CI 4.88 to 5.88). Inequalities were similar by gender and ethnicity. ${ }^{59}$ In contrast, Prazeres et al found no evidence of an association when screening EHRs. ${ }^{52}$

See table 2 for key results and quality assessments for these 13 studies, and online appendix 4 for more details on study characteristics.

Household composition $(\mathbf{n}=7)$ : Four studies measured household composition as living alone vs cohabiting and three studies measured it as living alone, living with various family members or living in other situations (including care homes).

Four cohort studies of older adults (50-84) reported mixed findings on the risk of living alone vs cohabiting. ${ }^{37454770}$ Two high-quality studies found living alone increased chances of multimorbidity vs living with others, ${ }^{37} 70$ for example, CantareroPrieto et al found living alone increased chances of multimorbidity by $20 \%$ (OR $1.20,95 \%$ CI 1.04 to $1.39, \mathrm{p}<0.05) .{ }^{70}$ Whereas two other studies-high and moderate quality - found no evidence, living alone was associated with multimorbidity incidence. ${ }^{45} 47$ Differences in study characteristics such as methods of ascertaining multimorbidity presence could not explain these mixed findings.

Of the three studies with alternative measures of composition, one moderate-quality cross-sectional study found that odds of multimorbidity were over 2 times greater if not living with children vs living with children (OR 2.11, 95\% CI 1.60 to 2.78; adjusted for age). ${ }^{61}$ Two further moderate-quality studies (one included solely older adults) found no evidence of any associations with multimorbidity when living alone was compared with living as a couple, with family/others or living in situations such as care homes. ${ }^{52} 60$

See table 3 for key results and quality assessments for these seven studies, and online appendix 4 for more details on study characteristics.

Household tenure $(n=4)$ : Findings from studies investigating tenure were mixed and hard to compare given different reference groups and comparators; two studies compared homeowners and non-homeowners, one compared renters with homeowners and one compared social housing residents with homeowners, private renters and subsidised housing residents. ${ }^{60656669}$ All four were of moderate quality. Lebenbaum et al found the odds of multimorbidity decreased by $18 \%$ for homeowners compared with nonhomeowners (OR $0.82,95 \%$ CI 0.78 to $0.87, \mathrm{p}<0.001)$ ) ${ }^{66}$ whereas Johnson-Lawrence et al reported 19\% higher odds for renters vs homeowners (OR 1.19, 95\% CI 1.15 to 1.24$).{ }^{69}$ In contrast, Schäfer et al-the only cohort study-found no evidence of an association between homeownership and count of conditions in older adults. ${ }^{60}$ One study found that, compared with social housing residents, homeowners and private renters had 17\% (OR 1.17, 95\% CI 1.11 to $1.24, \mathrm{p}=0.003$ ) and $19 \%$ (OR $1.19,95 \%$ CI 1.09 to $1.29, \mathrm{p}=0.041$ ) higher odds of multimorbidity, respectively. ${ }^{65}$ Differences in study characteristics could not explain mixed findings. 
Table 2 Key results and quality assessments for studies investigating household income $(n=13)$

\begin{tabular}{|c|c|c|c|c|c|c|c|c|}
\hline $\begin{array}{l}\begin{array}{l}\text { First author } \\
\text { (Year) }\end{array} \\
\begin{array}{l}\text { Household } \\
\text { income }\end{array}\end{array}$ & \multicolumn{4}{|l|}{ Key results } & \multicolumn{4}{|c|}{ Risk of Biasף } \\
\hline $\begin{array}{l}\text { Agborsangaya } \\
(2012)^{61}\end{array}$ & Yes & $\begin{array}{l}\text { OR } 2.39^{* *} \\
(1.72-3.33)\end{array}$ & $\begin{array}{l}\text { Annual household income } \\
<\$ 30 \mathrm{k} \text { vs } \geq \$ 100 \mathrm{k} C A D\end{array}$ & $\begin{array}{l}\text { Age, sex, education, living } \\
\text { with children }\end{array}$ & $\mathrm{H}$ & M & M & L \\
\hline $\begin{array}{l}\text { Agborsangaya } \\
(2013)^{62}\end{array}$ & Yes & $\begin{array}{l}\text { OR } 2.9 \\
(2.2-3.7)\end{array}$ & $\begin{array}{l}\text { Annual household income } \\
<\$ 30 \text { k vs } \geq \$ 100 \text { k CAD }\end{array}$ & $\begin{array}{l}\text { Age, sex, education, } \\
\text { obesity }\end{array}$ & $\mathrm{H}$ & H & $M$ & L \\
\hline $\begin{array}{l}\text { Hayek } \\
(2017)^{68}\end{array}$ & Yes & $\begin{array}{l}\text { PRR 1.7 } \\
(1.2-2.5 \\
p=0.005)\end{array}$ & $\begin{array}{l}\text { Monthly income } \leq \$ 2 \mathrm{k} \text { vs } \\
>\$ 4 \mathrm{k} \text { USD }\end{array}$ & Unclear & $U$ & H & $\mathrm{H}$ & U \\
\hline $\begin{array}{l}\text { Johnson- } \\
\text { Lawrence } \\
(2017)^{69}\end{array}$ & Yes & $\begin{array}{l}\text { OR } 1.45 \\
(1.38-1.53)\end{array}$ & $\begin{array}{l}\text { Lowest income tertile vs } \\
\text { highest }\end{array}$ & $\begin{array}{l}\text { Age, gender, ethnicity, } \\
\text { education, interview year, } \\
\text { region, marital status, last } \\
\text { doctor visit, employment, } \\
\text { home ownership }\end{array}$ & $U$ & M & $\mathrm{H}$ & L \\
\hline $\begin{array}{l}\text { Katikireddi } \\
(2017)^{39}\end{array}$ & Yes & $\begin{array}{l}\text { OR } 1.53 \\
(1.25-1.87 \\
p<0.05)\end{array}$ & $\begin{array}{l}\text { Lowest incomet tertile vs } \\
\text { highest }\end{array}$ & $\begin{array}{l}\text { Age, age }{ }^{2} \text {, age }{ }^{3} \text {, sex, } \\
\text { cohort, prior } \\
\text { multimorbidity, time } \\
\text { between waves and } \\
\text { sex }^{*} \text { cohort interaction }\end{array}$ & M & M & M & L \\
\hline $\operatorname{Ki}(2017)^{40}$ & Yes & $\begin{array}{l}\text { OR } 3.48^{*} \\
(3.20-3.78)\end{array}$ & $\begin{array}{l}\text { "Poor" (less than half the } \\
\text { median annual household } \\
\text { incomet) vs "non-poor" }\end{array}$ & No adjustment & U & H & M & H \\
\hline Laires $(2018)^{41}$ & Yes & $\begin{array}{l}\text { OR 2.16* } \\
(1.95-2.40)\end{array}$ & $\begin{array}{l}\text { Lowest incomet quintile } \\
\text { vs highest }\end{array}$ & No adjustment & $\mathrm{L}$ & H & M & H \\
\hline $\begin{array}{l}\text { Lebenbaum } \\
(2018)^{66}\end{array}$ & Yes & $\begin{array}{l}\text { OR } 0.57 \\
(0.52-0.62 \\
p<0.001)\end{array}$ & $\begin{array}{l}\text { Highest incomet quintile } \\
\text { vs lowest }\end{array}$ & $\begin{array}{l}\text { Age, age, }{ }^{2} \text { sex, marital } \\
\text { status, immigration status, } \\
\text { education, rurality, } \\
\text { homeownership, smoking, } \\
\text { alcohol use }\end{array}$ & $\mathrm{L}$ & M & $\mathrm{H}$ & L \\
\hline $\begin{array}{l}\text { Prazeres } \\
(2015)^{52}\end{array}$ & No & $\begin{array}{l}\text { OR 0.8§ } \\
(0.5-1.1 \\
p=0.182)\end{array}$ & $\begin{array}{l}\text { 'Some monthly income left } \\
\text { over' vs 'Not enough } \\
\text { monthly income to make } \\
\text { ends meet' }\end{array}$ & $\begin{array}{l}\text { Age, sex, marital status, } \\
\text { education, professional } \\
\text { status, residence area, } \\
\text { living arrangement }\end{array}$ & $\mathrm{H}$ & $M$ & L & L \\
\hline $\begin{array}{l}\text { Roberts } \\
(2015)^{53}\end{array}$ & Yes & $\begin{array}{l}\text { OR } 4.4 \\
(3.6-5.5)\end{array}$ & $\begin{array}{l}\text { Lowest income quintiles } \\
\text { vs highest }\end{array}$ & $\begin{array}{l}\text { Age, sex, household } \\
\text { education, Aboriginal } \\
\text { status, activity level } \\
\text { smoking, stress, blood } \\
\text { pressure, obesity }\end{array}$ & $\mathrm{H}$ & M & $\mathrm{H}$ & M \\
\hline $\begin{array}{l}\text { Schäfer } \\
(2012)^{60}\end{array}$ & Yes & $\begin{array}{l}-0.27 \\
\text { conditions } \\
(-0.47 \text { to } \\
-0.08 \\
p=0.005)\end{array}$ & $\begin{array}{l}\text { Change per unit on } \\
\text { incomet scale (one } \\
\text { unit=one of steps: } € 400 \text { to } \\
€ 1100 \text { to } € 3000 \text { to } € 8100 \\
\text { net income per month) }\end{array}$ & $\begin{array}{l}\text { Age, gender, marital } \\
\text { status, job autonomy, } \\
\text { household composition, } \\
\text { income }\end{array}$ & $\mathrm{H}$ & M & L & U \\
\hline Verest $(2019)^{59}$ & Yes & $\begin{array}{l}\text { OR } \\
5.36^{* \prime}+\dagger \\
(4.88-5.88)\end{array}$ & $\begin{array}{l}\text { "Lots of problems" } \\
\text { managing money vs "no } \\
\text { problems" }\end{array}$ & No adjustment & $\mathrm{H}$ & H & M & H \\
\hline
\end{tabular}

* OR calculated from data reported in paper.

tIncome equivalised to account for number and/or age of residents in household.

$\ddagger$ ‡ased on self-reported health data. Findings consistent across hospital and medication health data.

§Multimorbidity defined as $\geq 2$ chronic conditions.

१IH, High; M, Medium; L, Low; U, Unclear.

** Inequalities greater for ages $25-44$.

t+Inequalities greater for women and similar by ethnicity group. 
Table 3 Key results and quality assessments for studies investigating household composition $(n=7)$, household tenure $(n=4)$ and household rurality $(n=7)$, structured per social determinant

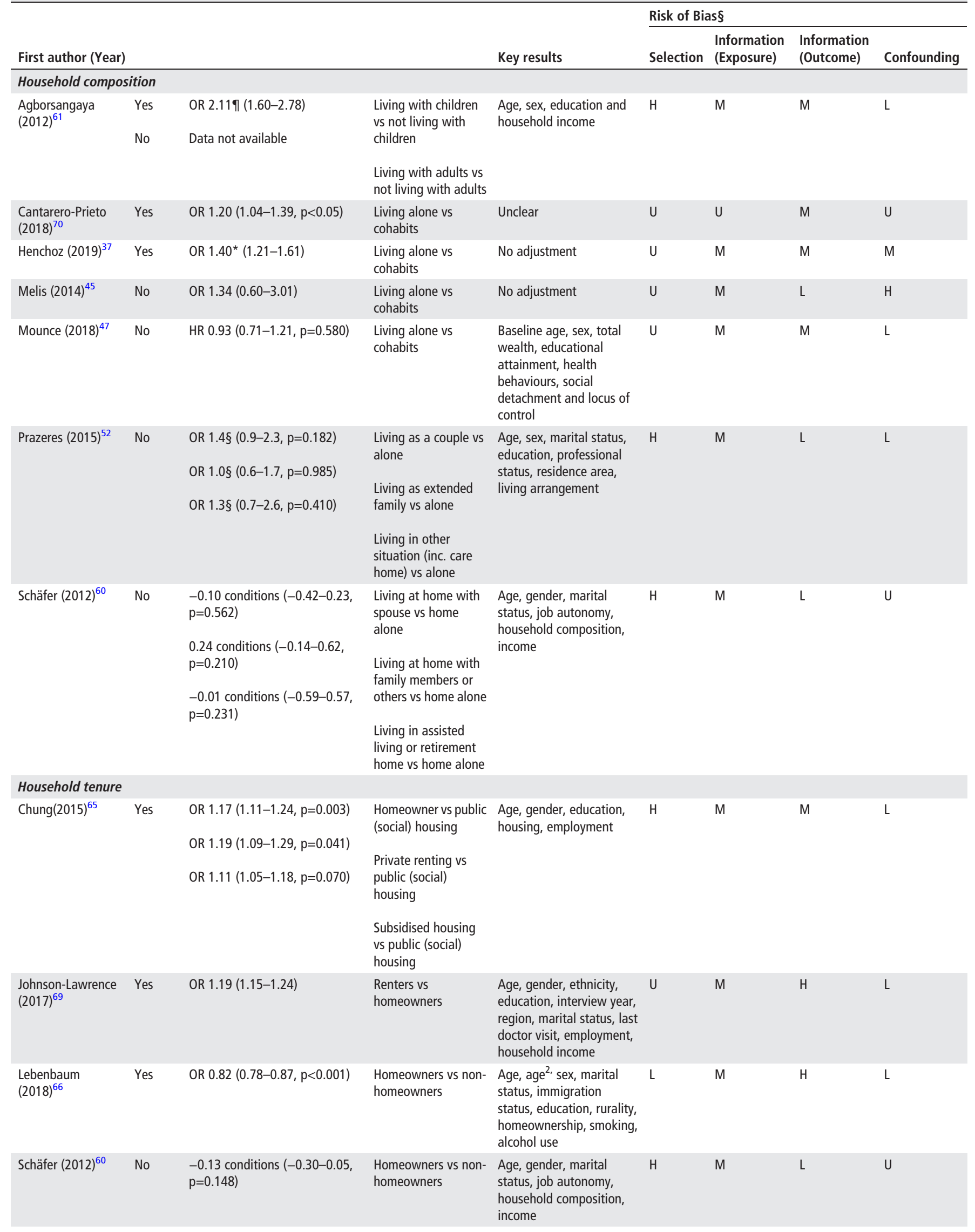




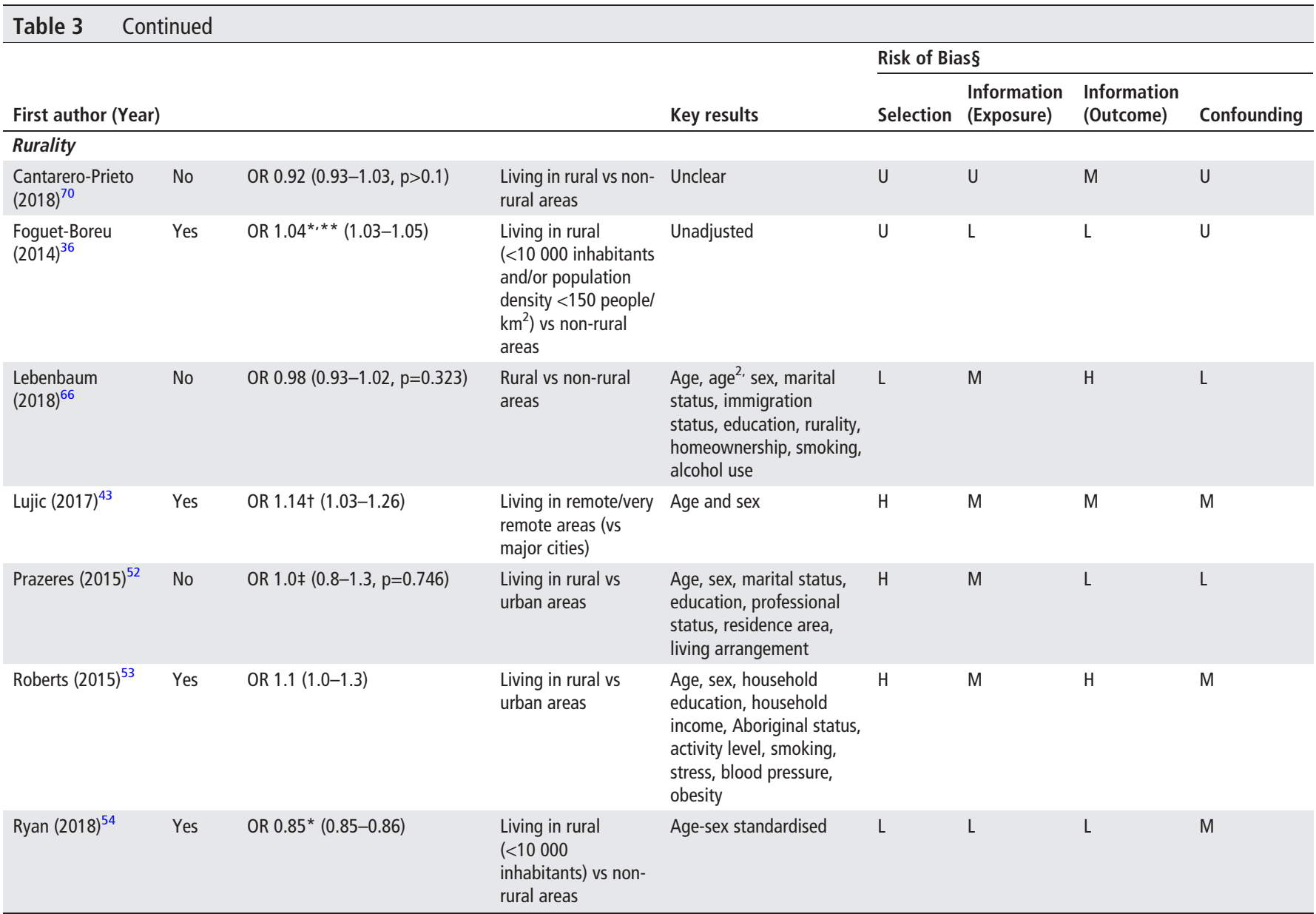

${ }^{*}$ OR calculated from data reported in paper.

tBased on self-reported health data. Findings consistent across hospital and medication health data.

$¥$ Multimorbidity defined as $\geq 2$ chronic conditions.

$\S \mathrm{H}$, High; M, Medium; L, Low; U, Unclear.

१Associations greater for $65+$.

**Inequalities similar with gender and greater $\geq 45$ years.

See table 3 for key results and quality assessments for these four studies, and online appendix 4 for more details on study characteristics.

Household determinants in childhood $(n=5)$ : Two studies examined associations between paternal social class at birth and multimorbidity. Findings were mixed. One higher quality study from Johnston et al found lower paternal social class at birth was associated with increased multimorbidity in middle age. ${ }^{72}$ Conversely, one study of lower quality reported no association. ${ }^{38}$

Two studies investigated associations between self-reported childhood financial hardships and multimorbidity and, again, findings were mixed. ${ }^{37} 71$ One higher quality study found no evidence of an association, ${ }^{37}$ whereas one fairly low-quality study found evidence that the number of chronic conditions for those reporting hardships was 1.19 times that of those not reporting hardships $(95 \%$ CI 1.07 to $1.32, \mathrm{p}<0.001){ }^{71}$

One further study, moderate in quality, found the odds of multimorbidity increased by $40 \%$ among those who had experienced household dysfunction during childhood (eg, parental divorce) in multivariate analyses (OR $1.4,95 \%$ CI 1.1 to $1.7, \mathrm{p}<0.05){ }^{56}$

Household primary language and education $(n=2)$ : One moderate-quality Australian study found associations between living in a household where English was the first language and multimorbidity prevalence differed depending on the source of health information. ${ }^{43}$ One lower quality study found higher odds of multimorbidity for participants living in households where no residents had completed high school compared with postsecondary school education (OR 1.8, 95\% CI 1.6 to 2.1, adjusting for age and sex). ${ }^{53}$

Household rurality $(n=7)$ : Studies that investigated associations between residing in rural vs non-rural/urban areas and multimorbidity reported mixed results. ${ }^{36} 4352-546670$ Only two studies were high quality and provided clear rurality definitions; these both suggested odds of multimorbidity decreased with increased rurality. ${ }^{3654}$ Conversely, two studies, one low ${ }^{53}$ and one moderate quality ${ }^{43}$ reported greater odds of multimorbidity with increased rurality. Three further studies found no evidence of any association. ${ }^{526670}$ Aside from study quality, differences in study characteristics could not explain these mixed findings.

See table 3 for key results and quality assessments for these seven studies, and online appendix 4 for more details on study characteristics.

Area-level socioeconomic deprivation $(n=17)$ : Studies that investigated how the socioeconomic situation of participants' residential area was associated with multimorbidity prevalence or incidence showed fairly consistent findings. 422343539424446 49-51545557636467 In general, multimorbidity was higher for participants residing in areas of greater deprivation than those 
living in more affluent areas. Odds of multimorbidity prevalence were $42 \%$ higher for participants residing in the most vs the least deprived areas when available data were pooled (OR 1.42, 95\% CI 1.41 to 1.42 ; figure 2). Differences in study quality could not explain differences in reported effect sizes across studies.

Four studies found inequalities with area-level deprivation were greater among women than men. ${ }^{35}{ }^{49-51}$ Only one low-quality study investigated how associations differed by ethnicity, and they found that inequalities were greater for Pacific vs Maori New Zealand residents. ${ }^{67}$

Trust in neighbours $(\mathbf{n}=1)$ : One fairly low-quality study found that participants who somewhat distrusted their neighbours had increased risk of developing multimorbidity within 11 years compared with those who strongly disagreed with the statement 'One cannot trust each other here' (RR $1.13,95 \%$ CI 1.03 to 1.23 ). ${ }^{58}$

Older adult populations and older adult sub-group analyses $(\mathbf{n}=21)$ : Nine studies included samples of adults solely aged 50 and over. ${ }^{373845-4750607071}$ Five of these examined associations between household composition and multimorbidity (results described above). ${ }^{3745476070}$ There were no differences in results for studies with older adults only compared with studies that included younger adults.

Thirteen studies examined whether associations differed with

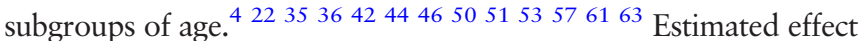
sizes were greater in younger compared with older adults for household income-for example, Roberts et al reported greater odds of multimorbidity amongst 35-49 year olds compared with over $65 \mathrm{~s}$ for those with the lowest income vs the highest (OR 7.5, 95\% CI 4.0 to 13.7 vs OR 2.5 , $95 \%$ CI 1.8 to 3.5 , respectively; see table 2$).{ }^{53}$ However, Agborsangaya et al (2012) found that associations between multimorbidity and not living with children (vs living with children) were greater for those $\geq 65$ years of age compared with those 25-44, adjusting for sex and household income (OR $8.45,95 \%$ CI 2.02 to 35.41 vs OR $2.00,95 \%$ CI 1.29 to 3.02 , respectively; see table 3). ${ }^{61}$ Ten studies found that differences in multimorbidity prevalence with area deprivation reduced in older

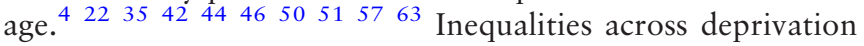

categories were greater in middle age for general

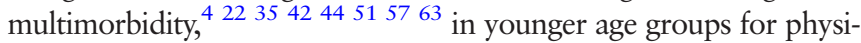
cal-mental multimorbidity ${ }^{4}{ }^{44}$ and mental-only multimorbidity, ${ }^{44}$ and in older age for physical-only multimorbidity. ${ }^{44}$ One study examining household rurality reported data showing similar associations in older vs young groups ${ }^{36}$ (see table 3 ).

Online appendix 2 outlines our full data extraction table for all 41 studies.

\section{DISCUSSION}

This is the first study to systematically review and appraise existing literature on associations between household and area-level $\mathrm{SDoH}$ and multimorbidity. Household income and area-level deprivation were the most explored SDs, and findings for these were fairly consistent; odds of multimorbidity were up to 4.4 times higher for those within the lowest level of household income (vs the highest), and prevalence was 1.4 times higher in the most vs the least deprived areas. Other household and arealevel SDoH have been underexplored.

\section{Possible explanations for our findings}

Previous research has proposed that household factors are often overlooked in studies exploring $\mathrm{SDoH}$, despite households (or families) influencing physical and mental health through various material and psychosocial factors. ${ }^{73-75}$

In this review, we identified seven studies that investigated household composition and four that investigated household tenure. Composition studies presented mixed results; living alone was associated with increased multimorbidity in two studies and not associated in four. These studies included different reference groups and comparators, making them hard to compare. For example, ill-health greatly drives care home admissions ${ }^{76}$ and therefore comparing 'living alone' with either 'not living alone' or 'living in a care home' would likely be comparing groups in different health, leading to differential associations between household composition

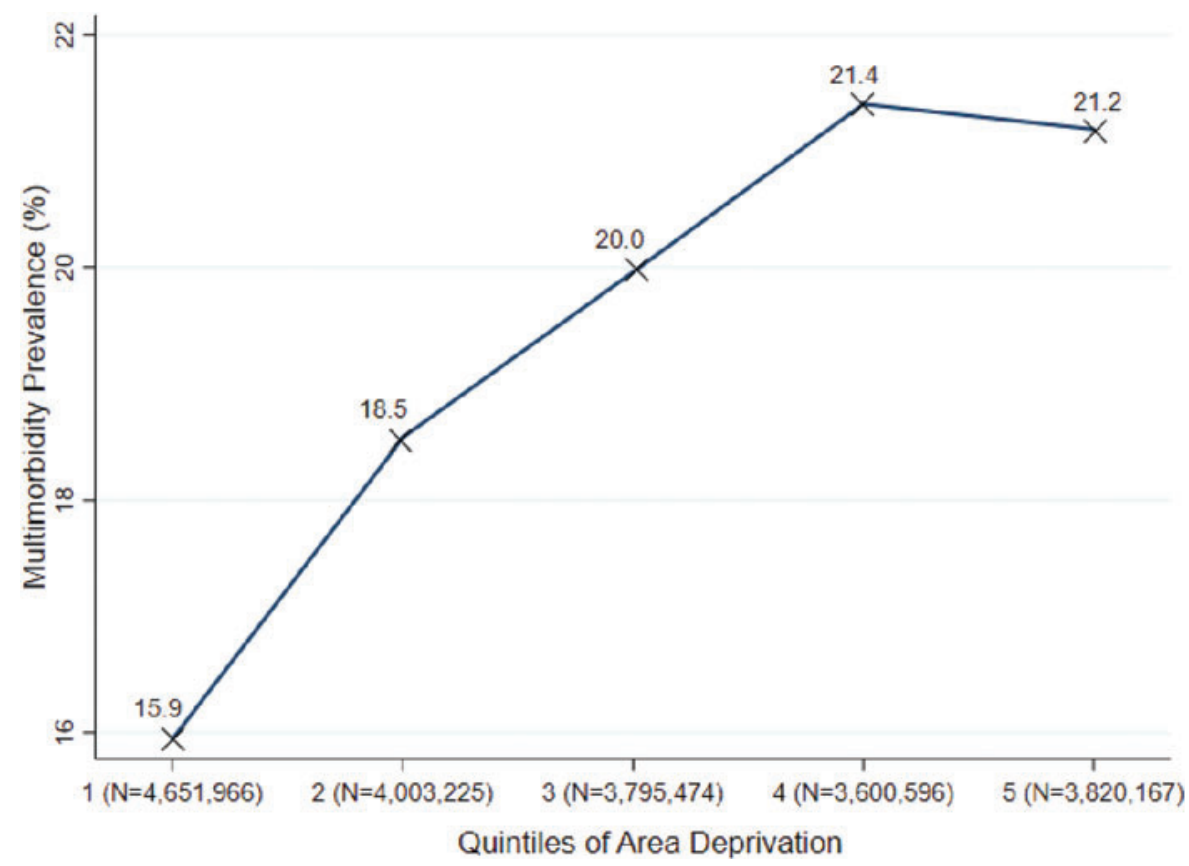

Figure 2 Multimorbidity prevalence with quintiles of area-level deprivation ( $1=$ least deprived and $5=$ most deprived). Calculations conducted by authors and based on available and relevant pooled data from $\mathrm{N}=7$ out of 17 studies. 
and multimorbidity. One further study found living with children (vs not) was associated with increased chances of multimorbidity, and this effect was greater for over $65 \mathrm{~s}$. Chronic illness may give rise to older individuals residing with family and may lead younger individuals unable to (or decide not to) have children. Interestingly, none of the included studies examining household composition adjusted for care provision, which can differ considerably for those living with a partner, family or alone ${ }^{77}$ and could plausibly influence the relationship between composition and multimorbidity. Further research should gather data on care provision and adjust accordingly. Unpicking whether social circumstances drive multimorbidity, or vice versa, also requires better designed longitudinal studies. This could aid the targeting of resources for prevention.

Studies investigating household tenure reported contradicting results; homeownership was associated with both increased and decreased chances of multimorbidity. Comparing these results was, again, complicated by different reference groups and comparators; however, study contexts may be more pertinent here. These studies were conducted in Hong Kong, Canada, USA and Germany. The degree of homeownership, and supply and conditions of social housing, may vary across these locations, for example, approximately $45 \%$ of the Hong Kong population lived in public housing in 2019 compared with $10 \%$ of the German population in 2017. ${ }^{78} 79$ This, plus other social circumstances, could profoundly influence the status and stigma associated with owning, renting, or residing in social housing across geographies and over time, differentially impacting health and associations between tenure and multimorbidity. ${ }^{80-82}$

A minority of studies examined whether associations differed by age, gender or ethnicity. Findings suggest women experience greater inequality in multimorbidity prevalence with area-level deprivation, in line with research highlighting an increase in life expectancy inequality for UK women. ${ }^{83}$ Prevalence with arealevel deprivation was also greater for younger populations for physical-mental multimorbidity, unsurprising given the consistently high prevalence of mental ill-health among young, deprived communities. ${ }^{84} 85$ This, however, suggests that studies excluding mental health conditions from multimorbidity definitions or specifying multimorbidity as specifically crossing physical and mental health may report different associations than studies not. Future research should consider physical and mental dimensions of multimorbidity and examine whether associations differ by key demographics. Further avenues for future research should also examine the main explanatory factors, for example, whether individual socioeconomic factors (such as education) or behavioural factors (such as tobacco use) confound or explain any observed associations. This could also aid the development of tailored prevention and intervention strategies.

Lack of consensus around a multimorbidity definition is a consistently raised issue. ${ }^{86}$ In this review, most studies defined multimorbidity as two or more chronic conditions (the most used definition in the literature ${ }^{86}$ ); however, several also used a cut-off point of three or more or a count of conditions. To ascertain the presence of multimorbidity, the included studies used either selfreported data, data from EHRs or a combination of the two. This hampered effective comparisons of study findings, yet we found no evidence suggesting differences in findings could be explained by differences in multimorbidity definitions or measurement methods. There was also no variation in determinants of multimorbidity by measurement methods used. Consistent definitions of multimorbidity and consistent methods for ascertaining its presence are needed to improve the comparability of findings.

\section{Strengths and limitations of this review}

Strengths of this paper include the systematic inclusion of household SDoH, which has captured studies missed by previous reviews, ${ }^{11}{ }^{15-18}$ and the careful assessment of each study for risk of four dimensions of bias using pre-specified criteria tailored to the study. Limitations include that we excluded the term 'comorbidity' and its linguistic variations from our search despite it being used interchangeably with 'multimorbidity' ${ }^{87}$ While this may have missed some relevant literature, a subsequent ad hoc search in MedLine, that included this term, did not identify any additional, relevant hits. A large proportion of our included studies were also identified via citation searching. We believe that this is an intrinsic issue when conducting these types of reviews; in the literature, $\mathrm{SDoH}$ are referred to by the determinant of interest (eg, 'rurality') and search strategies need to prespecify terms to search for these, potentially missing relevant studies. We also restricted our search to English-language publications and excluded studies conducted in low- and middleincome countries (LMICs) as the socioeconomic gradient in multimorbidity is reversed in LMICs. ${ }^{88}$

\section{Implications for policy, research and practice}

Household determinants of multimorbidity other than income are often overlooked. Given the comparatively large effect sizes for household compared with area-level $\mathrm{SDoH}$, our study suggests that strategies to tackle multimorbidity should consider household-level factors. There is also a need for additional studies in different geographical contexts to gain a better understanding of the role of household $\mathrm{SDoH}$ on multimorbidity. Policies aimed at reducing social inequalities could be important components of strategies to tackle multimorbidity.

\section{What is already known on this subject}

- Multimorbidity - the co-occurrence of multiple chronic conditions within the same individual-is influenced in extent and nature by social determinants of health (SDoH). Associations between individual SDoH, such as education and individual income, are well documented; prevalence is higher with lower education levels and income. However, no clear synthesis of household and arealevel social determinants of multimorbidity exists.

\section{What does this study add}

- We found that, aside from household income, household social determinants of multimorbidity are underexplored. Given the comparatively large effect sizes for household compared with area-level SDoH, our study suggests that strategies to tackle multimorbidity should consider household-level factors.

\section{Author affiliations}

${ }^{1}$ Department of Applied Health Research, University College London, London, UK

${ }^{2}$ Division of Psychiatry, University College London, London, UK

${ }^{3}$ London Boroughs of Camden and Islington, London, UK

${ }^{4}$ London School of Hygiene and Tropical Medicine, London, UK

${ }^{5}$ Camden and Islington NHS Foundation Trust, London, UK

Twitter Elizabeth Ingram @Lizzielngram11.

Contributors $\mathrm{El}, \mathrm{MG}, \mathrm{SH}, \mathrm{HM}, \mathrm{DO}$, and JS devised the research questions, methods and search strategy. EI, SL and SB screened the citations and implemented the search 
strategy. El developed the quality assessment criteria, and EI and SL critically appraised the included papers. El drafted the manuscript. All authors commented on drafts of the manuscript and agreed the decision to submit for publication.

Funding This study is independent research funded by the National Institute for Health Research School for Public Health Research (Grant Reference Number PD-SPH -2015-10025) and the National Institute for Health Research Applied Research Collaboration (ARC) North Thames. The views expressed in this publication are those of the authors and not necessarily those of the National Institute for Health Research or the Department of Health and Social Care.

Competing interests None declared.

Patient consent for publication Not required.

Provenance and peer review Not commissioned; externally peer-reviewed.

Supplemental material This content has been supplied by the author(s). It has not been vetted by BMJ Publishing Group Limited (BMJ) and may not have been peerreviewed. Any opinions or recommendations discussed are solely those of the author(s) and are not endorsed by BMJ. BMJ disclaims all liability and responsibility arising from any reliance placed on the content. Where the content includes any translated material, BMJ does not warrant the accuracy and reliability of the translations (including but not limited to local regulations, clinical guidelines, terminology, drug names and drug dosages), and is not responsible for any error and/ or omissions arising from translation and adaptation or otherwise.

Open access This is an open access article distributed in accordance with the Creative Commons Attribution 4.0 Unported (CC BY 4.0) license, which permits others to copy, redistribute, remix, transform and build upon this work for any purpose, provided the original work is properly cited, a link to the licence is given, and indication of whether changes were made. See: https://creativecommons.org/licenses/ by/4.0\%.

\section{ORCID iD}

Elizabeth Ingram http://orcid.org/0000-0002-0354-4551

\section{REFERENCES}

1 Pearson-Stuttard J, Ezzati M, Gregg EW. Multimorbidity: a defining challenge for health systems. Lancet Public Heal 2019;4:e599-e600.

2 Mercer S, Salisbury C, Fortin M. ABC of multimorbidity. Wiley Blackwell BMJ Books, 2014. Available http://as.wiley.com/WileyCDA/WileyTitle/productCd-EHEP003257.html

3 Fortin M, Soubhi H, Hudon C, et al. Multimorbidity's many challenges. Br Med J 2007;334:1016-17.

4 Cassell A, Edwards D, Harshfield A, et al. The epidemiology of multimorbidity in primary care: a retrospective cohort study. Br I Gen Pract 2018;68:e245-e251.

5 Kingston A, Robinson L, Booth $\mathrm{H}$, et al. Projections of multi-morbidity in the older population in England to 2035: estimates from the population ageing and care simulation (PACSim) model. Age Ageing 2018;47:374-80.

6 Mercer SW, Gunn J, Bower P, et al. Managing patients with mental and physical multimorbidity. BMJ 2012;345:11-12.

7 Sharp D, Lorenc A, Feder G, et al. 'Trying to put a square peg into a round hole': a qualitative study of healthcare professionals' views of integrating complementary medicine into primary care for musculoskeletal and mental health comorbidity. BMC Complement Altern Med 2018;18:290.

8 Dahlgren G, Whitehead M. Policies and strategies to promote social equity in health. 1991.

9 World Health Organisation. A conceptual framework for action on the social determinants of health. Geneva: World Health Organisation, 2010. ISBN 9789241500852. Available https://www.who.int/sdhconference/resources/

ConceptualframeworkforactiononSDH_eng.pdf (accessed 11 Sep 2019)

10 Kaplan GA. Part III summary: what is the role of the social environment in understanding inequalities in health? Adler NE, Marmot M, McEwen B, et al. Socioeconomic status and health in industrialized nations New York: Annals of the New York Academy of Sciences; 1999: 116-19.

11 Marengoni A, Angleman S, Melis R, et al. Aging with multimorbidity: a systematic review of the literature. Ageing Res Rev 2011;10:430-9.

12 Van den Akker M, Buntix F, Metsemakers JFM, et al. Multimorbidity in general practice: prevalence, incidence, and determinants of co-occurring chronic and recurrent diseases. J Clin Epidemiol 1998;51:367-75.

13 St Sauver JL, Boyd CM, Grossardt BR, et al. Risk of developing multimorbidity across all ages in an historical cohort study: differences by sex and ethnicity. BMJ Open 2015;5:2.

14 Rocca WA, Boyd CM, Grossardt BR, et al. The prevalence of multimorbidity in a geographically defined American population: patterns by age, sex, and ethnicity. BMJ Open 2015;89:1336-49.

15 The Academy of Medical Sciences. Multimorbidity: a priority for global health research. 2018. Available https://acmedsci.ac.uk/policy/policy-projects/multimorbidity (accessed 11 Sep 2019)

16 Violan C, Foguet-Boreu Q, Flores-Mateo G, et al. Prevalence, determinants and patterns of multimorbidity in primary care: a systematic review of observational studies. PLoS One 2014;9:3-11.
17 Pathirana TI, Jackson CA. Socioeconomic status and multimorbidity: a systematic review and meta-analysis. Aust N Z J Public Health 2018;42:186-94.

18 Northwood M, Ploeg J, Markle-Reid M, et al. Integrative review of the social determinants of health in older adults with multimorbidity. J Adv Nurs 2018;74:45-60.

19 Moher D, Liberati A, Tetzlaff J, et al. Preferred reporting items for systematic reviews and meta-analyses: the PRISMA statement. PLoS Med 2009;6:e1000097.

20 Marmot M. Fair society, healthy lives the marmot review. 2010. Available http://www. instituteofhealthequity.org/resources-reports/fair-society-healthy-lives-the-marmotreview/fair-society-healthy-lives-full-report-pdf.pdf

21 Moore KL, Boscardin WJ, Steinman MA, et al. Patterns of chronic co-morbid medical conditions in older residents of u.s. nursing homes: differences between the sexes and across the age span NIH public access. J Nutr Health Aging 2014;18:429-36.

22 Barnett K, Mercer SW, Norbury M, et al. Epidemiology of multimorbidity and implications for health care, research, and medical education: a cross-sectional study. Lancet 2012;380:37-43.

23 Newacheck PW, Stoddard JJ. Prevalence and impact of multiple childhood chronic illnesses. J Pediatr 1994;124:40-8.

24 Council on Social Determinants of Health C. A review of frameworks on the determinants of health canadian council on social determinants of health. 2015. Available http://ccsdh.ca/images/uploads/Frameworks_Report_English.pdf (accessed 28 Feb 2019)

25 Walker RJ, Smalls BL, Campbell JA, et al. Impact of social determinants of health on outcomes for type 2 diabetes: a systematic review. Endocrine 2014;47:29-48.

26 Duan-Porter W, Martinson BC, Greer N, et al. Evidence review: social determinants of health for veterans. J Gen Intern Med 2018;33:1785-95.

27 Nagata JM, Hernández-Ramos I, Kurup AS, et al. Social determinants of health and seasonal influenza vaccination in adults $\geq 65$ years: a systematic review of qualitative and quantitative data. BMC Public Health 2013;13:388.

28 Sterne J, Higgins JP, Elbers RG, et al. Risk of bias in non-randomized studies of interventions (ROBINS-1): detailed guidance. 2016. Available http://www.riskofbias. info (accessed 28 Oct 2019)

29 Viswanathan M, Patnode C, Berkman ND, et al. Assessing the risk of bias in systematic reviews of health care interventions. Methods guide for comparative effectiveness reviews. (Prepared by the scientific resource center under contract no. 290-20120004-C). Rockville, MD, 2017. Available https://effectivehealthcare.ahrq.gov/sites/ default/files/pdf/assessing-the-risk-of-bias-in-systematic-reviews-of-health-careinterventions-01_0.pdf (accessed 11 Sep 2019)

30 Centre for Reviews and Dissemination. Systematic reviews. CRD's guidance for undertaking reviews in health care. 2009. Available https://www.york.ac.uk/media/ crd/Systematic_Reviews.pdf (accessed 11 Sep 2019)

31 Wells G, Shea B, O'Connell D, et al. The Newcastle-Ottawa Scale (NOS) for assessing the quality of nonrandomised studies in meta-analyses. Published 2019. Available http://www.ohri.ca/programs/dinical_epidemiology/oxford.asp (accessed 28 Oct 2019)

32 McHugh ML. Interrater reliability: the kappa statistic. Biochem Medica 2012;22:276-82. (accessed 11 Sep 2019).

33 O'Halloran J, Miller GC, Britt H. Defining chronic conditions for primary care with ICPC-2. Fam Pract 2004:21:381-6.

34 Charlton J, Rudisill C, Bhattarai N, et al. Impact of deprivation on occurrence, outcomes and health care costs of people with multiple morbidity. J Health Serv Res Policy 2013:18:215-23.

35 Violán C, Foguet-Boreu Q, Roso-Llorach A, et al. Burden of multimorbidity, socioeconomic status and use of health services across stages of life in urban areas: a cross-sectional study. BMC Public Health 2014;14:530.

36 Foguet-Boreu Q, Violan C, Roso-Llorach A, et al. Impact of multimorbidity: acute morbidity, area of residency and use of health services across the life span in a region of south Europe. BMC Fam Pract 2014;15:55.

37 Henchoz Y, Seematter-Bagnoud L, Nanchen D, et al. Childhood adversity: a gateway to multimorbidity in older age? Arch Gerontol Geriatr 2018;80:31-7.

38 Humphreys J, Jameson K, Cooper C, et al. Early-life predictors of future multi-morbidity: results from the Hertfordshire cohort. Age Ageing 2018;47:474-8.

39 Katikireddi SV, Skivington K, Leyland AH, et al. The contribution of risk factors to socioeconomic inequalities in multimorbidity across the lifecourse: a longitudinal analysis of the twenty-07 cohort. BMC Med 2017;15:152.

$40 \mathrm{Ki} \mathrm{M}$, Lee YH, Kim Y-S, et al. Socioeconomic inequalities in health in the context of multimorbidity: a Korean panel study. Zeeb H, ed. PLoS One 2017; 12:e0173770.

41 Laires PA, Perelman J. The current and projected burden of multimorbidity: a cross-sectional study in a Southern Europe population. Eur J Ageing 2019;16:181-92.

42 Li J, Green M, Kearns B, et al. Patterns of multimorbidity and their association with health outcomes within Yorkshire, England: baseline results from the Yorkshire Health Study. BMC Public Health 2016;16:649.

43 Lujic S, Simpson JM, Zwar N, et al. Multimorbidity in Australia: comparing estimates derived using administrative data sources and survey data. Islam FMA, ed. PLoS One 2017; 12:e0183817.

44 McLean G, Gunn J, Wyke S, et al. The influence of socioeconomic deprivation on multimorbidity at different ages: a cross-sectional study. Br J Gen Pract 2014;64:440-7.

45 Melis R, Marengoni A, Angleman S, et al. Incidence and predictors of multimorbidity in the elderly: a population-based longitudinal study. PLoS One 2014;9:e103120. 
46 Moin JS, Moineddin R, Upshur REG. Measuring the association between marginalization and multimorbidity in Ontario, Canada: a cross-sectional study. J Comorbidity 2018:8:2235042X18814939.

47 Mounce LTA, Campbell JL, Henley WE, et al. Predicting incident multimorbidity. Ann Fam Med 2018;16:322-9.

48 Nielsen CR, Halling A, Andersen-Ranberg K. Disparities in multimorbidity across Europe: findings from the SHARE survey. Eur Geriatr Med 2017;8:16-21.

49 Orueta JF, García-Álvarez A, Alonso-Morán E, et al. Socioeconomic variation in the burden of chronic conditions and health care provision: analyzing administrative individual level data from the Basque Country, Spain. BMC Public Health 2013;13:870.

50 Orueta JF, Nuño-Solinís R, García-Alvarez A, et al. Prevalence of multimorbidity according to the deprivation level among the elderly in the Basque Country. BMC Public Health 2013:13:918.

51 Orueta JF, García-Álvarez A, García-Goñi M, et al. Prevalence and costs of multimorbidity by deprivation levels in the basque country: a population based study using health administrative databases. PLoS One 2014;9:e89787.

52 Prazeres F, Santiago L. Prevalence of multimorbidity in the adult population attending primary care in Portugal: a cross-sectional study. BMJ Open 2015; 5:e009287. (accessed 11 Sep 2019).

53 Roberts KC, Rao DP, Bennett TL, et al. Prevalence and patterns of chronic disease multimorbidity and associated determinants in Canada. Heal Promot Chronic Dis Prev Canada Res Policy Pract 2015;35:87-94. Available http://www.ncbi.nlm.nih.gov/ pubmed/26302227 (accessed July 17, 2019)

54 Ryan BL, Bray Jenkyn K, Shariff SZ, et al. Beyond the grey tsunami: a cross-sectional population-based study of multimorbidity in Ontario. Can J Public Heal 2018;109:845-54

55 Salisbury C, Johnson L, Purdy S, et al. Epidemiology and impact of multimorbidity in primary care: a retrospective cohort study. Br J Gen Pract 2011;61:e12-21.

56 Sinnott C, Mc Hugh S, Fitzgerald AP, et al. Psychosocial complexity in multimorbidity: the legacy of adverse childhood experiences. Fam Pract 2015;32:269-75.

57 Stanley J, Semper K, Millar E, et al. Epidemiology of multimorbidity in New Zealand: a cross-sectional study using national-level hospital and pharmaceutical data. BMJ Open 2018;8:e021689.

58 Tomasdottir MO, Sigurdsson JA, Petursson H, et al. Does "existential unease" predict adult multimorbidity? Analytical cohort study on embodiment based on the Norwegian HUNT population. BMJ Open 2016;6:e012602.

59 Verest WJGM, Galenkamp H, Spek B, et al. Do ethnic inequalities in multimorbidity reflect ethnic differences in socioeconomic status? The HELIUS study. Eur J Public Health 2019; February.

60 Schäfer I, Hansen H, Schön G, et al. The influence of age, gender and socio-economic status on multimorbidity patterns in primary care. first results from the multicare cohort study. BMC Health Serv Res 2012;12:89.

61 Agborsangaya CB, Lau D, Lahtinen M, et al. Multimorbidity prevalence and patterns across socioeconomic determinants: a cross-sectional survey. BMC Public Health 2012;12:201.

62 Agborsangaya $C B$, Ngwakongnwi $E$, Lahtinen $M$, et al. Multimorbidity prevalence in the general population: the role of obesity in chronic disease clustering. BMC Public Health 2013;13:1161.

63 Arbelle JE, Chodick G, Goldstein A, et al. Multiple chronic disorders - health care system's modern challenge in the Maccabi health care system. Isr J Health Policy Res 2014;3:29.

64 Bähler C, Huber CA, Brüngger B, et al. Multimorbidity, health care utilization and costs in an elderly community-dwelling population: a claims data based observational study. BMC Health Serv Res 2015;15:23.

65 Chung RY, Mercer S, Lai FTT, et al. Socioeconomic determinants of multimorbidity: a population-based household survey of Hong Kong Chinese. Marengoni A, ed. PLOS One 2015; 10:e0140040.
66 Lebenbaum M, Zaric GS, Thind A, et al. Trends in obesity and multimorbidity in Canada. Prev Med (Baltim) 2018:116:173-9.

67 Stokes T, Azam M, Doolan Noble F. Multimorbidity in Māori and Pacific patients: crosssectional study in a Dunedin general practice. J Prim Health Care 2018;10:39.

68 Hayek S, Ifrah A, Enav T, et al. Correlates, and time trends of multiple chronic conditions among Israeli adults: estimates from the Israeli national health interview survey, 2014-2015. Prev Chronic Dis 2017;14:E64.

69 Johnson-Lawrence V, Zajacova A, Sneed R. Education, race/ethnicity, and multimorbidity among adults aged 30-64 in the national health interview survey. SSM Popul Health 2017;3:366-72.

70 Cantarero-Prieto D, Pascual-Sáez M, Blázquez-Fernández C. Social isolation and multiple chronic diseases after age 50: a European macro-regional analysis. LathamMintus K, ed. PLoS One 2018; 13:e0205062.

71 Tucker-Seeley RD, Li Y, Sorensen G, et al. Lifecourse socioeconomic circumstances and multimorbidity among older adults. BMC Public Health 2011;11.

72 Johnston MC, Black C, Mercer SW, et al. Impact of educational attainment on the association between social class at birth and multimorbidity in middle age in the Aberdeen children of the 1950s cohort study. BMJ Open 2019:9:e024048.

73 McNeill T. Family as a social determinant of health: implications for governments and institutions to promote the health and well-being of families. Healthc Q 2010;14Spec No: 60-67:60-7

74 Ross CE, Mirowsky J, Goldsteen K. The impact of the family on health: the decade in review. Journal of Marriage and the Family 1990;52:1059-1078.

75 Vaezghasemi M, Ng N, Eriksson M, et al. Households, the omitted level in contextual analysis: disentangling the relative influence of households and districts on the variation of BMI about two decades in Indonesia. Int J Equity Health 2016;15:102.

76 Bowman C, Whistler J, Ellerby M. A national census of care home residents. Age Ageing 2004:33:561-6.

77 Hellström Y, Hallberg IR. Determinants and characteristics of help provision for elderly people living at home and in relation to quality of life. Scand J Caring Sci 2004;18:387-95

78 South China Morning Post. How Hong Kong's public housing system works: costs, waiting times and sales. 2019. Available https://www.scmp.com/news/hong-kong /society/article/2182106/why-public-housing-shortfall-will-remain-thorn-hong-kongs

79 Eurostat. Housing statistics. 2020. Available https://ec.europa.eu/eurostat/statisticsexplained/index.php/Housing_statistics\#Type_of_dwelling (accessed 7 Feb 2020)

80 Shaw M. Housing and public health. Annu Rev Public Health 2004;25:397-418.

81 Reeves A, Clair A, Mckee M, et al. Reductions in the United Kingdom's government housing benefit and symptoms of depression in low-income households. Am J Epidemiol 2016;184:421-9.

82 Clair A, Hughes A. Housing and health: new evidence using biomarker data. J Epidemiol Community Health 2019;73:256-62.

83 Marmot M. Health equity in England: the Marmot review 10 years on. BMJ 2020; m693.

84 Kessler RC, Berglund P, Demler O, et al. Lifetime prevalence and age-of-onset distributions of DSM-IV disorders in the national comorbidity survey replication. Arch Gen Psychiatry 2005;62:593-602.

85 Bond L, Kearns A, Mason P, et al. Exploring the relationships between housing, neighbourhoods and mental wellbeing for residents of deprived areas. BMC Public Health 2012:12:48

86 Johnston MC, Crilly M, Black C, et al. Defining and measuring multimorbidity: a systematic review of systematic reviews. Eur J Public Health 2019:29:182-9.

87 van den Akker M, Buntinx F, Knottnerus JA. Comorbidity or multimorbidity. Eur J Gen Pract 1996;2:65-70.

88 Kunna R, San Sebastian M, Stewart Williams J. Measurement and decomposition of socioeconomic inequality in single and multimorbidity in older adults in China and Ghana: results from the WHO study on global AGEing and adult health (SAGE). Int J Equity Health 2017;16:79. 